\title{
DISPLACEMENT-BASED RAPID SEISMIC ASSESSMENT PROCEDURE FOR BUILDING STRUCTURES
}

\author{
H.H. TSANG ${ }^{*}$, R.K.L. SU ${ }^{* *}$, N.T.K. LAM ${ }^{* * *}$ and S.H. LO ${ }^{* *}$ \\ ${ }^{*}$ Department of Civil Engineering, \\ Chu Hai College of Higher Education, \\ Hong Kong, China: \\ tsang@chuhai.edu.hk \\ ${ }^{* *}$ Department of Civil Engineering, \\ The University of Hong Kong, \\ Hong Kong, China \\ ${ }^{* * *}$ Department of Civil and Environmental Engineering, \\ The University of Melbourne. \\ Melbourne, Australia
}

\begin{abstract}
Seismic assessment of building structures can be a very involved process. This paper presents a simplified and rational manual procedure for rapid predictions of maximum inter-storey drift demand in tall buildings, which does not require frame analysis nor finite element analysis to be carried out and can be expedited by the use of a spreadsheet program. The methodology comprises a series of stages, beginning with the development of an elastic design response spectrum (or a uniform hazard spectrum) for specifying the level of seismic hazard, followed by the inclusion of a site-specific factor which represents the effects of soil amplification. The maximum seismic inter-storey drift demand of the building is then predicted from the displacement response spectrum for a given height and the estimated lateral natural periods of the building. The proposed manual procedure can be further developed to cater for limited ductility demand behaviour in the building. The predicted drift demand is an important indicator of potential seismic damage (risk), and may be utilized for the rapid assessment of damage and loss (cost) for considered earthquake scenarios.
\end{abstract}

Keywords: design spectrum, displacement, inter-storey drift, nonlinear site response, seismic demand.

\section{Introduction}

It has been recognized that urban areas located at long distances from tectonic plate margins, broadly categorized as regions of low-to-moderate seismicity, may be significantly affected by earthquake tremors. They are typically characterised by a high density of population, high concentrations of high-rise buildings, complex infrastructure, and a very large proportion of reclaimed land. Therefore, although the seismic hazard of those cities remains moderate, the seismic risk, in terms of damage potential to structures, loss of lives, assets, businesses and services, can be very high. Such situation can be observed in many major Asian cities, such as Hong Kong, Beijing, Singapore and Kuala Lumpur. Citing Hong Kong as an example, due to the constraint of the natural topography and a high demand of housing as well as commercial needs, reclamation of land from the sea has been widely practiced in the territory. 
Traditionally high-rise buildings are assumed to perform better during earthquakes because of their longer natural period and therefore reduced seismic force demand. However, recent studies show that high-rise buildings with some structural irregularities can potentially face problems under ground shaking due to high seismic demand levels at locations of sudden stiffness changes ( $\mathrm{Su}$ et al., 2002). A high-rise building designed for wind would generally have large stiffness but would not necessarily have the desired ductility and redundancy for resisting seismic cyclic loads. On the other hand, low to medium rise buildings are designed to resist a smaller wind force and they may be more susceptible to seismic loading. Typical buildings falling into this category are schools, hospitals and fire stations. Buildings on soft ground may be especially vulnerable. Authorities around the world now recognise that buildings and infrastructure in low-tomoderate seismic regions require a minimum level of protection against future earthquakes.

Seismic assessment of building structures can be a very involved process in countries of high seismicity where dynamic analyses of the soil sedimentary layers are often required for determining the potential amplification characteristics of the site. The site amplification study is then followed by the dynamic analysis of the building involving finite element modelling. In countries of low-to-moderate seismicity which do not already have earthquake design provisions in its building regulations, certain stake holders and governance authorities are very conscious of the potential seismic risks of its infrastructure. Many are prepared to address these risks and undertake retrofitting where necessary. Assessing the vulnerability of its existing building stocks is the first step. Assessment studies can be of a very large scale involving the entire city. Clearly, it is too costly and unrealistic to carry out intensive analyses involving finite element modelling of the site, or the building, or both given that a large number of sites and buildings may have to be covered with limited time and resources. In this paper, a simplified and rational manual procedure (Tsang et al., 2009) has been presented to serve this purpose. The procedure does not require frame analysis nor finite element analysis to be carried out and can be expedited by the use of a spreadsheet program.

Unlike the United States as well as other developed countries of high seismicity, many countries in the low-to-moderate seismic regions do not have a seismic design code specifying the format of design spectrum. In the light of this, the procedure begins with the development of simplified soil response spectra based on a regional elastic design response spectrum (or uniform hazard spectrum) for rock sites. This step can allow detailed site-specific information to be incorporated into the seismic hazard model. The key components of this procedure are soil damping ratio formula, period shift ratio formula and nonlinear site response formula. All these formulae are comprehensive in nature, with appropriate consideration of the level of shaking, impedance contrast between soil and bedrock and the plasticity of the soil layer. This technique is considered particularly suitable for low-to-moderate seismic regions, where there is a scarcity of strong motion records. 
The paper further deals with the estimations of the lateral natural periods of the building. By combining the period estimation with the seismic hazard prediction model, maximum seismic inter-storey drift demand of the building can be estimated, based initially on elastic response considerations. The assumptions of linear elastic behaviour will not severely limit the scope of applications of the procedure in areas of low-to-moderate seismic demands where the majority of the buildings will not be excited into the inelastic range. In such conditions, the procedure can be used to identify buildings, or parts of the building, which have the potential of experiencing yielding and hence requiring followup investigations with more rigorous tools. Notwithstanding, the proposed manual procedure can be further developed to cater for limited ductility demand behaviour in the building. The predicted drift demand is an important indicator of potential seismic damage (risk), and may be utilized for the rapid assessment of damage and loss (cost) for considered earthquake scenarios. This paper is aimed at a broad international readership interested in adopting a methodology to estimate the seismic drift demand of tall buildings in metropolitan cities located in low-to-moderate seismic regions.

\section{Design Spectrum for Rock Sites}

For regions where a seismic design code is available, design spectrum can be derived based on the specified format and hazard level. In Hong Kong, where a seismic design code does not exist, design spectrum has to be developed fundamentally from probabilistic seismic hazard assessment (PSHA) for different probability levels. The well-established technique for PSHA was developed originally by Cornell (1968). This commonly adopted methodology incorporates the influence of all potential sources of earthquakes and their corresponding mean activity rates. As this approach may be difficult to apply in regions lacking sufficient information concerning the geological setting, active faults, and so forth, an alternative procedure, namely the direct amplitudebased $(D A B)$ approach, has been developed to assess site-specific seismic hazard (Tsang and Chandler, 2006). This approach has been shown to be as reliable as the conventional approach. There are a number of distinctive advantages, for example, it is not necessary to characterize any seismic sources that are basically a highly subjective exercise. Also, "site-specific" and "event-specific" ground motion model can be adopted.

Using $D A B$ approach, the probability of exceedance (PE) of a certain level of shaking can be obtained. Also, response spectral values have been computed for the whole period range of engineering interest, to form a site-specific uniform hazard spectrum (UHS), that can be used for the typical bedrock condition in Hong Kong (Chandler et al., 2006).

An alternative approach is to construct the design response spectrum using simplified expressions for the spectrum corner periods and the maximum response spectral parameters (Lam et al., 2000a), whilst the later can be calculated from the component attenuation model (CAM) (Lam et al., 2000b). CAM is a generic response spectral attenuation model that comprises a series of component factors: source factor, path factors and crustal factors. It provides quick estimates for maximum response spectral parameters with a reasonable accuracy using hand-calculation formulae. It is believed to be particularly useful for areas where local strong motion data are lacking for satisfactory 
empirical modelling. With the use of CAM, a tri-linear design response spectrum can easily be constructed.

\section{Design Spectrum accounting for Nonlinear Site Effects}

Soil overlying bedrock can have significant effects on both the amplitude and frequency characteristics of earthquake ground shaking. This has been widely observed and reported in recent damaging earthquakes such as those in Northridge, California (1994), Kobe, Japan (1995), and Turkey (1999). One key contributory factor for earthquake-induced damage is the lengthening of the site natural period due to nonlinearity of site response under moderate to strong levels of bedrock shaking. This feature is considered potentially critical for long period structures, such as tall buildings or medium to long span bridges. The period shift effect is accompanied by an increase in energy dissipation, as larger shear strain is developed in the soil layer. The frequency content can influence the damage potential of earthquake ground motions in both the elastic and inelastic ranges. If the natural period of structure is close to the site natural period, then, significant amplification of the structural response would occur due to resonance. In this section, a simple technique for constructing a site-specific displacement response spectrum $(R S D)$ has been described (Tsang et al., 2006a).

\subsection{Nonlinear Site Response Estimation}

The key element of this model is the use of the spectral ratio $(S R)$ at the site natural period $T_{g}$ (considering the period-shift effect). It is defined as the ratio between the maximum spectral velocity (or displacement) of soil spectrum $\left(R S V_{\max }\right.$ or $R S D_{\max }$ at period $T_{g}$ ) and the spectral velocity (or displacement) of bedrock spectrum at period $T_{g}$ $\left(R S V_{T g}\right.$ or $\left.R S D_{T g}\right)$, and has been proposed to be composed of two components: the peak velocity (or displacement) ratio $(P V R$ or $P D R)$ and a resonance factor $f(\alpha)$ :

$$
\begin{gathered}
S R=\frac{R S V_{\max }}{R S V_{T g}}=P V R \cdot f(\alpha) \\
\text { where } \quad P V R=\frac{2 \alpha}{1+\alpha} \sqrt{\frac{\beta}{1-R^{4} \beta^{4}}}
\end{gathered}
$$

which is defined as the ratio between the peak ground velocity on the surface of the soil sediment and that on the bedrock surface and

$$
f(\alpha)=\alpha^{0.3} \leq 2.3
$$

which is used to scale up the nonlinear PVR, for response spectrum modelling. Eqs. (1) and (2) were developed theoretically based on basic wave theories and the concept of multiple reflections of seismic waves within the soil sediments. The accuracy of these two formulae has been demonstrated by a comprehensive comparison with results obtained from equivalent linear shear wave analyses and data recorded during the 1994 Northridge earthquake (Tsang et al., 2006a). 


$$
\alpha=\frac{\rho_{R} V_{R}}{\rho_{S} V_{S}} \text { is the impedance ratio }
$$

in which, $\rho$ and $V$ are the weighted-average of the density and the shear wave velocity (SWV) of the respective layers, and the subscripts R and S represent the rock and soil layers, respectively. It is noted that, theoretically, the averages of the seismic velocity (V) and density $(\rho)$ should be determined independently, instead of averaging the seismic impedance.

$R$ is the reflection coefficient, which describes the amplitude ratio of the upwardly propagating reflected wave and the downwardly propagating incident wave within the soil layer, and is expressed by:

$$
R=\frac{1-\alpha}{1+\alpha}
$$

The parameter $\beta$ is related to the energy dissipation within soil layer, and is defined as:

$$
\beta=\exp (-\pi \zeta)
$$

where $\zeta$ is the soil damping ratio and can be estimated by Tsang et al. (2006b):

$$
\begin{aligned}
\zeta & =12.5+6.5 \log \left(R_{\gamma} \lambda \psi\right)-0.13 P I \\
\text { where } \psi & =\frac{R S V_{T g}}{V_{S}}=\frac{R S D_{T g}}{H} \frac{\pi}{2}
\end{aligned}
$$

$R_{\gamma}$ is the ratio of the effective shear strain to maximum shear strain, which has been empirically found to vary between about 0.5 to 0.7 ( 0.6 has been used in this study). $P I$ is the plasticity index of soil material (in \%). $H$ is the total thickness of soil layer.

The reduction factor $\lambda$ is needed to account for the bedrock rigidity effect:

$$
\lambda=\frac{\alpha}{1+\alpha} \sqrt{\frac{1-\beta^{4}}{1-R^{4} \beta^{4}}}
$$

Eq. (7) may be bounded by a "practical" minimum damping ratio $\zeta_{p i}$ and an upper bound damping ratio $\zeta_{u b}$ :

$$
\begin{aligned}
& \zeta_{p i}(\%)=2.5+0.03 \cdot \operatorname{PI}(\%) \leq 6.8 \\
& \zeta_{u b}(\%)=17.5-0.07 \cdot \operatorname{PI}(\%) \geq \zeta_{p i}
\end{aligned}
$$

Finally, the period shift ratio can be estimated by Tsang et al. (2006b):

$$
\frac{T_{g}}{T_{i}}=1+R_{\gamma} \lambda \psi \mu
$$

The parameter $\mu$, termed the "Plasticity Factor", is used to allow for different PI, as shown in Table 1. 
Table 1. The Plasticity Factor $\mu$ [refer Eq. (12)] for different plasticity indices (PI).

\begin{tabular}{lllll}
\hline Plasticity Index PI (\%) & 0 & 15 & 30 & 50 \\
\hline Plasticity Factor $\mu$ & 1.6 & 0.9 & 0.4 & 0.2 \\
\hline
\end{tabular}

\subsection{Site-specific Displacement Response Spectrum (RSD) Modelling}

The simple procedure for constructing soil RSD has been described as follows:

(i) Obtain the basic parameters from normal site investigation: initial SWV $V_{S i}$ (or $S P T$ " $N$ " value) and $P I$, thickness $H$ of the soil layer; bedrock SWV $V_{R}$. Initial site natural period $T_{i}$ can then be computed by Eq. (13). $R S V_{T i}$ or $R S D_{T i}$ can be obtained from rock response spectrum.

$$
T_{i}=\frac{4 H}{V_{S, i}}
$$

(ii) Calculate the soil damping ratio, by Eq. (7), with $\lambda=1 ; R S V_{T g}=R S V_{T i}$ (or $R S D_{T g}=$ $\left.R S D_{T i}\right), V_{S}=V_{S i}$; and then the damping factor $\beta$, by Eq. (6), and the reduction factor $\lambda$, by Eq. (9).

(iii) Calculate the degraded soil $V_{S}$, by Eq. (14), with the consideration of the following relationship:

$$
\frac{V_{S}}{V_{S i}}=\frac{T_{i}}{T_{g}}
$$

The actual shifted site natural period $T_{g}$, can then be computed by Eq. (12), using the degraded soil $V_{S} \cdot R S V_{T g}$ (or $R S D_{T g}$ ) can then be obtained from rock response spectrum. (It is noted that if $R S D_{T g}$ is used in the calculation, it is not required to compute the degraded soil $V_{S}$.)

(iv) The impedance ratio $\alpha$ [Eq. (4)], the reflection coefficient $R$ [Eq. (5)] and the soil damping ratio [Eq. (7)], together with the damping factor $\beta$ [Eq. (6)] can then be calculated.

(v) The $R S V_{\max }$ or $R S D_{\max }$ can be computed by Eqs. (1) - (3), and the two parameters can also be linked by Eq. (15):

$$
\frac{R S D_{\max }}{R S V_{\max }}=\frac{T_{g}}{2 \pi}
$$

(vi) The idealised bi-linear soil RSD can be constructed for any given site (Tsang et al., 2006a), as demonstrated in Figure 1. 


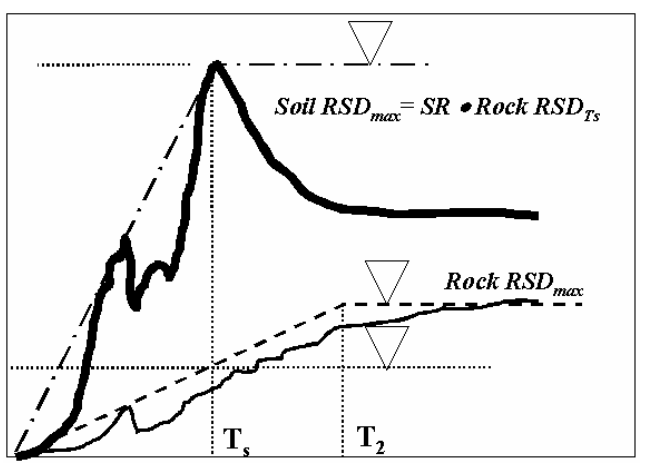

Fig. 1. Idealised bi-linear soil displacement response spectrum (RSD) model (Tsang et al., 2006a).

\subsection{Application for Hong Kong Soil Sites}

Two representative soil and reclamation (fill) sites from different locations of Hong Kong territory have been selected for illustration. Central (CL) site is situated on Hong Kong Island and Tseung Kwan O (TKO) site is situated in Kowloon. The basic parameters required by Step One, as described in the previous section, are summarized in Table 2. The typical bedrock $V_{R}$ of $2000 \mathrm{~m} / \mathrm{s}$ (Chandler et al., 2006) has been assumed for both sites, and the density ratio $\rho_{\mathrm{R}} / \rho_{\mathrm{s}}$ has been assumed as 1.3 and 1.6, respectively. $R S V_{T i}$ can be obtained from the rock response spectra, and is given in Table 2.

Table 2. Basic parameters for Central (CL) and Tseung Kwan O (TKO) sites.

\begin{tabular}{llllll}
\hline Site & $H(\mathrm{~m})$ & $V_{S, i}(\mathrm{~m} / \mathrm{s})$ & $P I(\%)$ & $T_{i}(\mathrm{~s})$ & $R S V_{T i}(\mathrm{~mm} / \mathrm{s})$ \\
\hline CL & 60 & 317 & 15 & 0.76 & 78 \\
TKO & 63 & 214 & 15 & 1.18 & 77 \\
\hline
\end{tabular}

Following the steps described, site-specific $R S D$ can be obtained and are shown in Figure 2, together with that for a typical rock site in Hong Kong. Significant different results between the three sites address the importance of soil amplification.

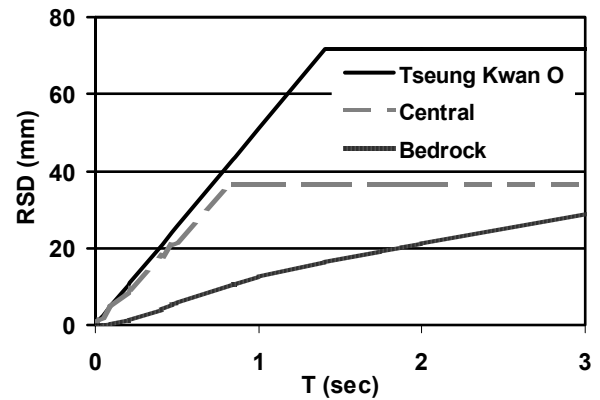

Fig. 2. Displacement response spectra $(R S D)$ for a typical bedrock site, Central (CL) and Tseung Kwan O (TKO) soil sites in Hong Kong. 


\section{Estimation of Lateral Building Periods}

Dynamic responses of building structures depend primarily on the fundamental natural periods of the buildings. Extensive investigations of natural periods of buildings in Hong Kong by ambient vibration tests (AVT) were conducted by Su et al. (2003). The natural periods of buildings with heights ranging from $50 \mathrm{~m}$ to $415 \mathrm{~m}$ were measured. Linear regression analysis revealed that for buildings of conventional structural forms above 50 $\mathrm{m}$ in height, the fundamental lateral building period may be estimated by Eq. (16):

$$
T_{1}=\frac{H_{b}}{72}
$$

which gives a high correlation (R-square) value of 0.85 . Further, the periods of the second $T_{2}$ and the third $T_{3}$ modes can be estimated by Sheikh (2005):

$$
T_{2}=\frac{T_{1}}{4} \quad \text { and } \quad T_{3}=\frac{T_{1}}{8}
$$

It has been recognised that effective (true) lateral periods of buildings associated with large vibration amplitudes are typically longer than the AVT results, due to, for instance, flexural cracking of elements of concrete and masonry structures, flexibility of foundations, and so forth. It is recommended that a period shift factor of 1.5 should be applied for $\mathrm{PE}=10 \%$ in 50 years (Su et al., 2008). It is noted that larger factor should be applied for smaller PE as period shift is dependent on the level of ground shaking.

Two Hong Kong buildings, namely the Swire Building (SB) and Typical Harmony Block Building (THB), were chosen in this study. SB is a 15-storey reinforced concrete (RC) building, in which the lateral force-resisting system consists of $\mathrm{RC}$ moment-resisting frames attached to concrete core walls at the centre and four walls at the edges. THB is a 41-storey RC shear-wall building. The structure has four similar wings, which are symmetrically arranged in the longitudinal and lateral directions with a cruciform configuration and are attached to the central core of the building.

The lateral periods of the two buildings were measured directly by AVT. The effective lateral vibration periods (considering the period shift factor of 1.5 to account for the stiffness degradation effects) have been calculated and tabulated in Table 3.

Table 3. The effective (true) lateral periods of Swire Building (SB) and Typical Harmonic Block Building (THB).

\begin{tabular}{lllll}
\hline Building & $H_{b}(\mathrm{~m})$ & $T_{1}(\mathrm{~s})$ & $T_{2}(\mathrm{~s})$ & $T_{3}(\mathrm{~s})$ \\
\hline SB & 51.25 & 1.07 & 0.27 & 0.13 \\
THB & 112.7 & 2.35 & 0.59 & 0.29 \\
\hline
\end{tabular}

\section{Seismic Inter-storey Drift Estimation}

Displacement, as opposed to force, has been recognised as the preferred criterion for seismic design and performance assessment of buildings and infrastructure. Displacement-based $(D B)$ approach has attracted worldwide attention in research over the past decade. Current codified response spectrum models developed within the context of 
the conventional force-based $(F B)$ procedures would translate into unrealistic displacement demand predictions, particularly in the long period range for high-rise buildings (Priestley and Kowalski, 2000).

A simplified theoretical framework for modelling maximum seismic inter-storey drift angle $\theta_{\max }$ of multi-storey buildings has been developed in Sheikh (2005), considering the first three lateral modes of vibration. Firstly, the average seismic inter-storey drift angle $\theta_{\text {avg }}$ can be defined as (with the contribution from the fundamental mode only):

$$
\theta_{\text {avg }}=\frac{\Delta_{\text {roof }}}{H_{b}}
$$

where $\Delta_{\text {lroof }}$ is the lateral displacement at roof level (due to the fundamental vibration mode only), and can be related to the response spectral displacement at the fundamental modal period $R S D_{1}$ (termed the effective displacement) by the multiplier $\lambda_{\text {avg }}$ :

$$
\lambda_{\text {avg }}=\frac{\Delta_{\text {lroof }}}{R S D_{1}}
$$

Secondly, maximum inter-storey drift angle $\theta_{1 \max }$ (due to the fundamental vibration mode only) may be related to the average drift angle by the drift multiplier $\lambda_{1}$ as:

$$
\lambda_{1}=\frac{\theta_{1 \max }}{\theta_{\text {avg }}}
$$

Then, maximum drift angle can be related to $\theta_{1 \max }$, with consideration of the first three lateral modes of vibration, by the drift multiplier $\lambda_{2}$ as:

$$
\lambda_{2}=\frac{\theta_{\max }}{\theta_{1 \max }}
$$

Hence, by Eqs. (19) - (22), the maximum drift $\theta_{\max }$ can be related to $R S D_{1}$ by:

$$
\theta_{\max }=\lambda_{1} \lambda_{2} \lambda_{\text {avg }} \frac{R S D_{1}}{H_{b}}
$$

Definition of drift angles has been shown in Figure 3.
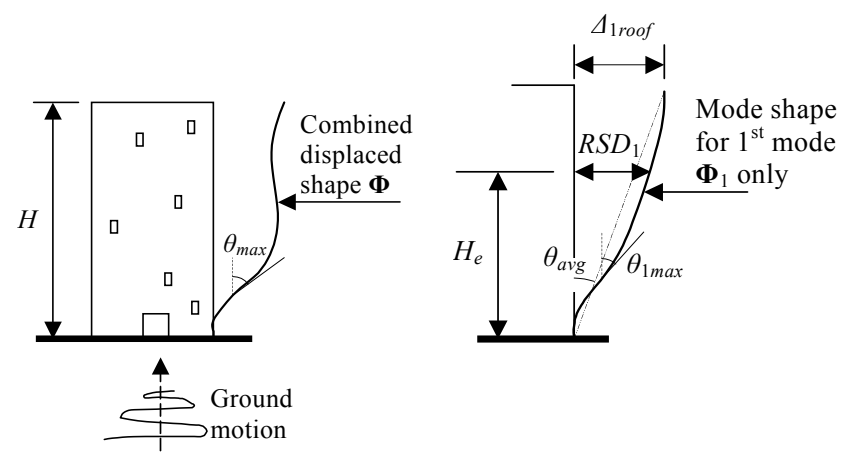

Fig. 3. Definition of drift angles $\theta_{\text {avg }}, \theta_{1 \max }$ and $\theta_{\max }$. 
Parametric studies (Sheikh, 2005) indicate that both drift multipliers $\lambda_{\text {avg }}$ and $\lambda_{1}$ can be assumed equal to 1.5 , with appropriate conservatism. The recommended $\lambda_{\text {avg }}$ value is based on taking the reciprocal of the participation factor for the fundamental mode of vibration which is typically of the order of $0.6-0.7$. The recommended $\lambda_{1}$ value is based on the shape of a parabola.

For the higher-mode drift multiplier $\lambda_{2}$, it is dependent on the input earthquake ground motion, in particular on the ratio of $R S D_{2} / R S D_{1} . R S D_{2}$ is the seismic response spectral displacement at the second modal period, and $\lambda_{2}$ can be estimated by:

$$
\lambda_{2}=0.35+2.0 \frac{R S D_{2}}{R S D_{1}} \geq 1.0
$$

Regarding the third mode of vibration, it is found that the sensitivity of $\lambda_{2}$ to changes of ratio of $R S D_{3} / R S D_{2}$ is very small. Depending on the magnitude of the maximum drift demand, modification for potential inelastic effects may be implemented. The effective (or equivalent) damping ratio of the entire building may be expressed by Eq. (25):

$$
\beta_{\text {eff }}(\%)=1000 \times \theta_{\max }
$$

Note that response spectra developed in previous sections are based on $5 \%$ damped design level. The demand response spectrum should then be modified, for the effective damping ratio, using the damping modification factor $(D M F)$, according to Eurocode 8:

$$
D M F=\sqrt{\frac{10}{5+\beta_{\text {eff }}}}
$$

As Eq. (25), and hence Eq. (26), depends on the estimation of $\theta_{\max }$ [Eq. (23)], iteration process is required. It has been found that three such iterations are sufficient for the result to converge. The maximum inter-storey drift $\theta_{\max }$ estimated for the hazard level of $\mathrm{PE}=$ $10 \%$ and $2 \%$ in 50 years for the two buildings and located on the three sites have been shown in Figure 4.

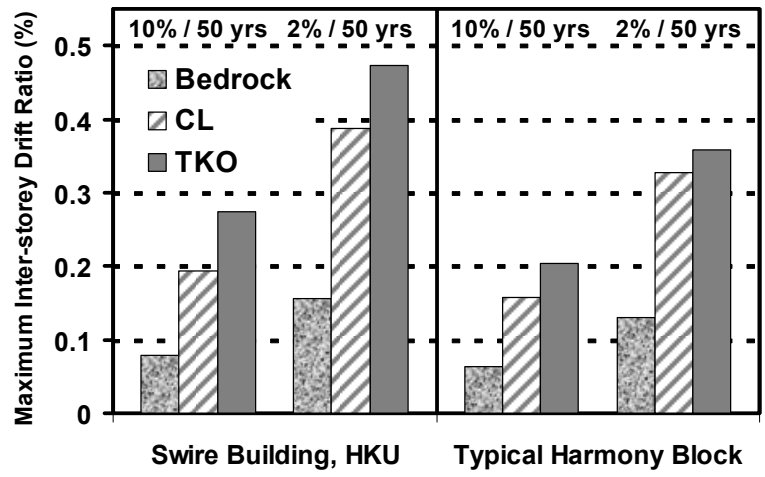

Fig. 4. Maximum inter-storey drift angles $\theta_{\max }$ estimated for the hazard level of $\mathrm{PE}=10 \%$ and $2 \%$ in 50 years. 
The maximum drift is found to vary significantly between different sites, which demonstrates the highly site-dependent nature in estimating seismic drift demand of buildings. For the hazard level of $\mathrm{PE}=10 \%$ in 50 years, the maximum drift estimated for $\mathrm{SB}$, situated on the deep soil site in TKO is found to be $0.27 \%$. In addition, for the hazard level of $\mathrm{PE}=2 \%$ in 50 years, the maximum drift estimated for $\mathrm{SB}$ and THB, situated on deep soil sites as in CL and TKO is found to be in the order of $0.45 \%$ and $0.35 \%$, respectively.

\section{Conclusions}

A new methodology for the rapid prediction of maximum inter-storey drift demand in tall buildings has been presented, which comprises a series of stages:

(i) Seismic hazard assessment for rock sites has been presented, using a newly developed probabilistic method, termed the Direct Amplitude-Based (DAB) approach.

(ii) A site-specific factor has been introduced to allow for nonlinear soil amplification. The comprehensive formulae for computing this factor take into account the potential nonlinear behaviour of soil materials, shear stiffness of soil and bedrock materials and the plasticity of the soil. A site-specific displacement response spectrum can then be constructed. Two soil sites in Hong Kong have been chosen for demonstration purposes. Significantly different results between the two sites address the importance of soil amplification.

(iii) Lateral vibration periods of buildings can be estimated based on building heights. Localised formula employed indicates the importance of considering local practice of building design and construction. Two buildings of different heights have been chosen for demonstration and comparison.

(iv) The maximum inter-storey drift ratio can be estimated based on the derived displacement demand spectra, building heights and lateral periods. The drift demand can be modified for different structural damping levels, depending on the magnitude of the maximum drift demand.

(v) The technique has been illustrated using two buildings, with the consideration of three different site conditions. The maximum drift is found to vary significantly between different sites, which demonstrates the highly site-dependent nature in estimating seismic drift demand of buildings.

\section{References}

A. M. Chandler, N. T. K. Lam and H. H. Tsang, J. Asian Earth Sci. 27(6), 892 (2006).

C. A. Cornell, Bull. Seismol. Soc. Am. 58, 1583 (1968).

N. T. K. Lam, J. L. Wilson, A. M. Chandler and G. L. Hutchinson, Earthquake Eng. Struct. Dynam. 29, 1491 (2000a).

N. T. K. Lam, J. L. Wilson, A. M. Chandler and G. L. Hutchinson, Earthquake Eng. Struct. Dynam. 29, 1457 (2000b).

M. J. N. Priestley and M. J. Kowalski, Bull. New Zeal. Natl. Soc. Earthquake Eng. 33(4), 421 (2000).

M. N. Sheikh, Seismic assessment of buildings in Hong Kong with special emphasis on displacement-based approaches, PhD Thesis, The University of Hong Kong (2005).

R. K. L. Su, A. M. Chandler, J. H. Li and N. T. K. Lam, Struct. Eng. Mech. 14(3), 287 (2002).

R. K. L. Su, A. M. Chandler, P. K. K. Lee, A. P. To and J. H. Li, Transactions of Hong Kong Institution of Engineers 10(2), 17 (2003). 
R. K. L. Su, N. T. K. Lam and H. H. Tsang, Electronic J. Struct. Eng. 8, 110 (2008).

H. H. Tsang and A. M. Chandler, Bull. Seismol. Soc. Am. 96(2), 392 (2006).

H. H. Tsang, A. M. Chandler and N. T. K. Lam, Earthquake Eng. Struct. Dynam. 35(9), 1053 (2006a).

H. H. Tsang, A. M. Chandler and N. T. K. Lam, Earthquake Eng. Struct. Dynam. 35(15), 1925 (2006b).

H. H. Tsang, R. K. L. Su, N. T. K. Lam and S. H. Lo, The Structural Design of Tall and Special Buildings, 18(4), 427 (2009). 